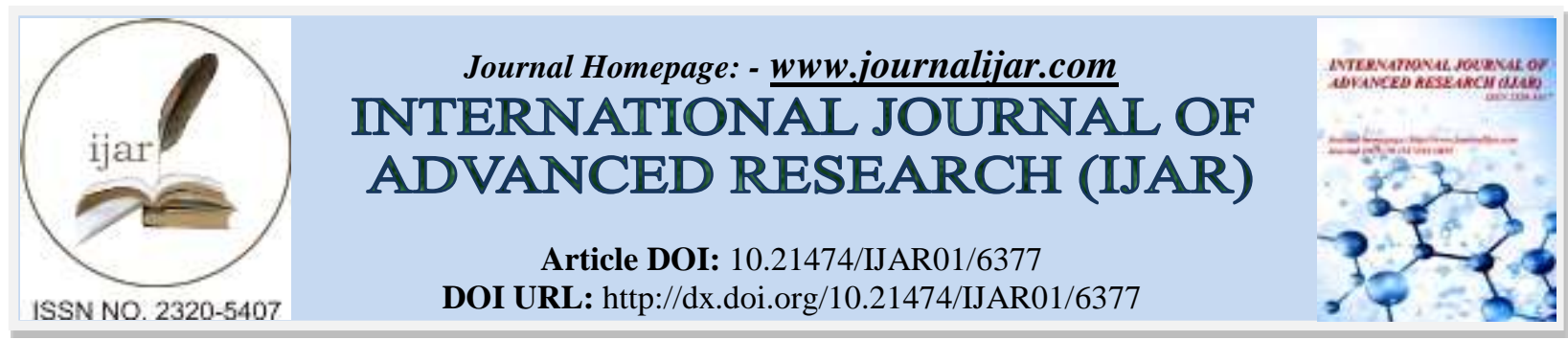

RESEARCH ARTICLE

\title{
DISCRIMINANT SCORE (D SCORE) A MODEL TO PREDICT LUNG CANCER BASED ON THE EXPRESSION STUDY OF PANEL OF GENES AND MICRORNAS IN THE PLASMA.
}

Dr. Hussain Abady Aljebori.

MB ChB (Baghdad), MSc Path (Glasgow UK) and Arab Board of surgical pathology and PhD molecular pathology.

\section{Manuscript Info}

Manuscript History

Received: 22 November 2017

Final Accepted: 24 December 2017

Published: January 2018

Key words:-

Lung cancer, Plasma cell free nuclei acid, qRT-PCR, D-Score.

\section{Abstract}

Background: Lung cancer is the leading cause of death from cancer worldwide in adult, and accounting for (13\%) of all newly diagnosed cancer cases. Inspite of that there is no effective method for screening of highly susceptible groups such as chronic heavy smokers. The present study depends on expression of panel of genes and microRNAs that are involved or the result of lung cancer formation in blood samples taken from cancer and non-cancer lung cases to design a discrimination score that can exclude or predict lung cancer. Objective: Is to design a discrimination score (D score) to predict lung cancer in blood samples based of expression study of a panel of genes and microRNAs. Patients and Method: A case control study on expression of P53, KRAS, c-MYC, and Her-2/neu genes, microRNAs 21, 34a, 92, and 98 in blood samples taken from patients positive and negative for lung cancer. Results: The discrimination score (D score) was obtained per a mathematical equation for each sample. According to the value of discrimination score: any new case with a discrimination score (D score) more than 0.0 is considered as a lung cancer and the higher the result the more we are confident the case is lung cancer. Any case with discrimination score below 0.0 is considered as a benign lung condition, and the lower the result the more we are confident the case is a benign condition.

Copy Right, IJAR, 2018,. All rights reserved.

\section{Introduction:-}

Lung cancer is the commonest cause of death from cancer in male worldwide and constituting a major health problem because of widespread tobacco smoking [1]. Its peak of incidence in 50s and 60s and rarely before 40s, although it is more common in males, but its incidence is rising in females because of increasing smoking habit among young ladies [1]. According to Iraqi Ministry of health statistics 2009, lung cancer constituted $8 \%$ of total cancer and was the second cause of death after heart diseases [2]. Inspite of this worldwide problem there is no efficient method for screening of susceptible groups. Most cases were diagnosed at late stages and were beyond radical treatment. Nowadays, the current laboratory methods for diagnosis depend on sputum cytopathology for malignant cells which is not a sensitive method and can't be adopted as a screening method [3]. Invasive procedures such as fine needle aspiration cytopathology (FNAC) under sonar or CT scan, core needle biopsy, bronchoscopy with bronchial wash, bronchial brush, bronchoscopic biopsy and/or open biopsy are highly sensitive and specific for detecting lung cancer but they not suitable as screening methods because they are invasive procedures and most of patient are severely ill and can't tolerate them $[4,5,6]$. The present study was designed depending on the new 
advances in molecular and cancer pathology. Particularly the use of cell free nuclei acid in the plasma as tumor markers $[7,8,9]$. The present work was based on studying the expression of genes and microRNAs that are the cause or the result of lung cancer formation, namely P53 [10, 11], KRAS [12], c-MYC [13], Her-2/neu [14], microRNAs-21 [16, 17], 34a [18], 92 [19], and 98 [20] genes in the plasma of patient diagnosed as cancer and benign lung diseases in order to obtain a discrimination score for differentiating malignant from benign lung conditions.

\section{Study objectives:-}

Determination by quantitative realtime RT-PCR of expression of mRNAs and microRNAs in question in blood samples taken from patient with lung cancer and benign conditions. Also obtaining a discrimination score (D score) to differentiate lung cancer from benign lung conditions.

\section{Patients:-}

Patients were recruited at the Surgical Thoracic Unit in Al-Yarmouk Teaching Hospital during the period from March 2015 to April 2017.The study was a prospective case-control study in which 60 patients were participated. They were divided into two groups of 30 patients with and 30 without lung cancer proved by cytopathology and/or histopathology. The study was carried out at postgraduate laboratory / Al-mustansiriyah College of Medicine. The work was ethically approved by the internal commity of ethics of Al-mustansiriyah college of medicine and a signed consent was taken from each participant patient before taking the samples.

\section{Methods:-}

Extraction of mRNAs and microRNAs: Under aseptic technique $10 \mathrm{ml}$ of venous blood aspirated from each patient the first $2 \mathrm{ml}$ was discarded and remaining blood was introduced into labelled nuclease free EDTA containing tube. The plasma was obtained from each sample by centrifugation. Plasma samples were taken for RNA extraction using mirVana ${ }^{\mathrm{TM}}$ miRNA Isolation Kit, with phenol according to manufacturer instructions [21].

qRT-PCR amplification of mRNAs: The obtained total RNAs then reversed transcribed into cDNA using HighCapacity RNA-to-cDNA ${ }^{\text {TM }}$ Kit from Applied Biosystem according to manufacturer protocol [22]. The cDNAs of mRNAs then amplified by realtime PCR using Applied Biosystems ${ }^{\text {TM }}$ SYBR ${ }^{\text {TM }}$ Green PCR Master Mix according to manufacturer instructions [23] and Primers for forty cycles figure-2. The primers were synthesized by Applied Biosystem and designed according to NCBI/primer-BLAST system. Glyceraldehyde 3-phosphate dehydrogenase (GAPDH) was choosed as a housekeeping gene [24]. At the end of cycles, the $\mathrm{Ct}$ values for each study mRNAs and housekeeping genes are recorded for each sample for further analysis.

qRT-PCR amplification of microRNAs: The cDNA from obtained microRNAs then amplified by realtime PCR using primers and TaqMan® MicroRNA Master Mix II, no UNG Kit from Applied Biosystems for microRNAs according to manufacturer instruction [25]. the has-mir-16 were adopted as housekeeping genes for micro RNAs [26]. The thermal profile consisting of forty cycles. At the end of cycles, the Ct values are recorded for all studied microRNAs and housekeeping gene obtained from each studied sample for further studies.

\section{Statistical analysis:-}

Analysis of clinical findings such as age and gender of patients.

Analysis of cytopathological and/or histopathologic findings.

Analysis of findings from realtime PCR machine Agilent technology MxPro 3000P software [27] which are: Amplification curves, $\mathrm{Ct}$ values, dissociation curves, and consolidated results.

The $\Delta \mathrm{Ct}$ and $\Delta \Delta \mathrm{Ct}$ for each gene were calculated according to the equations:

The $\Delta \mathrm{Ct}$ of target gene $=[\mathrm{Ct}$ of target gene $-\mathrm{Ct}$ of housekeeping gene $]$.

The $\Delta \mathrm{Ct}$ of control gene $=[\mathrm{Ct}$ of control gene $-\mathrm{Ct}$ of housekeeping gene $]$. Expression of gene $=\left(2^{-\Delta \Delta \mathrm{C}}\right)$, the result of expression $=2^{-\Delta \Delta \mathrm{C}}$.

$\Delta \Delta \mathrm{C}=\{[\mathrm{Ct}$ of target gene $-\mathrm{Ct}$ of housekeeping gene] - [Ct of control $-\mathrm{Ct}$ of housekeeping gene] $\}$ [27]

Discrimination Score (D) equation was designed according to SPSS for items analysis. D score was calculated for each studied sample whether malignant or benign according to the following equation designed according to SPSS item analysis software $[28,29,30,31]$, the D score equation is: 
D score $=-219.7+(-0.342 \times$ CompCt_P53 $)+(3.322 \times$ Comp Ct_KRAS $)+(-0.296 \times$ Comp Ct_c-MYC $)+(1.016 \times$ CompCT_Her2neu $)+(0.346 \times$ CompCT_mir21 $)+(-24.026 \times$ CompCT_mir34a $)+(0.67 \times$ CompCT_mir92 $)+$ (0.388 x CompCT_mir98).

Sample is considered malignant when D score is $>0.0$, and the higher the positive value of D score the more confident the tested specimen is really a cancer case.

Sample is considered benign when D score is $<0.0$, the lower the negative value of D score the more confident the tested specimen is not a cancer case.

\section{Results:-}

Lung cancer was more common in males than females with ratio of $2 / 1$. Non-small cell (NSCLC) was the more common than small cells lung cancer (SCLC) constituting 24/30 (80\%) and 6/30 (20\%) respectively. Squamous cell bronchogenic carcinoma was the commonest type of lung cancer accounting for 19/30 (63.34\%), adenocarcinoma 4/301(3.33\%), large cell carcinoma 1/30 (3.33\%), and small cell lung carcinoma 6/30 (20\%), figure-1.

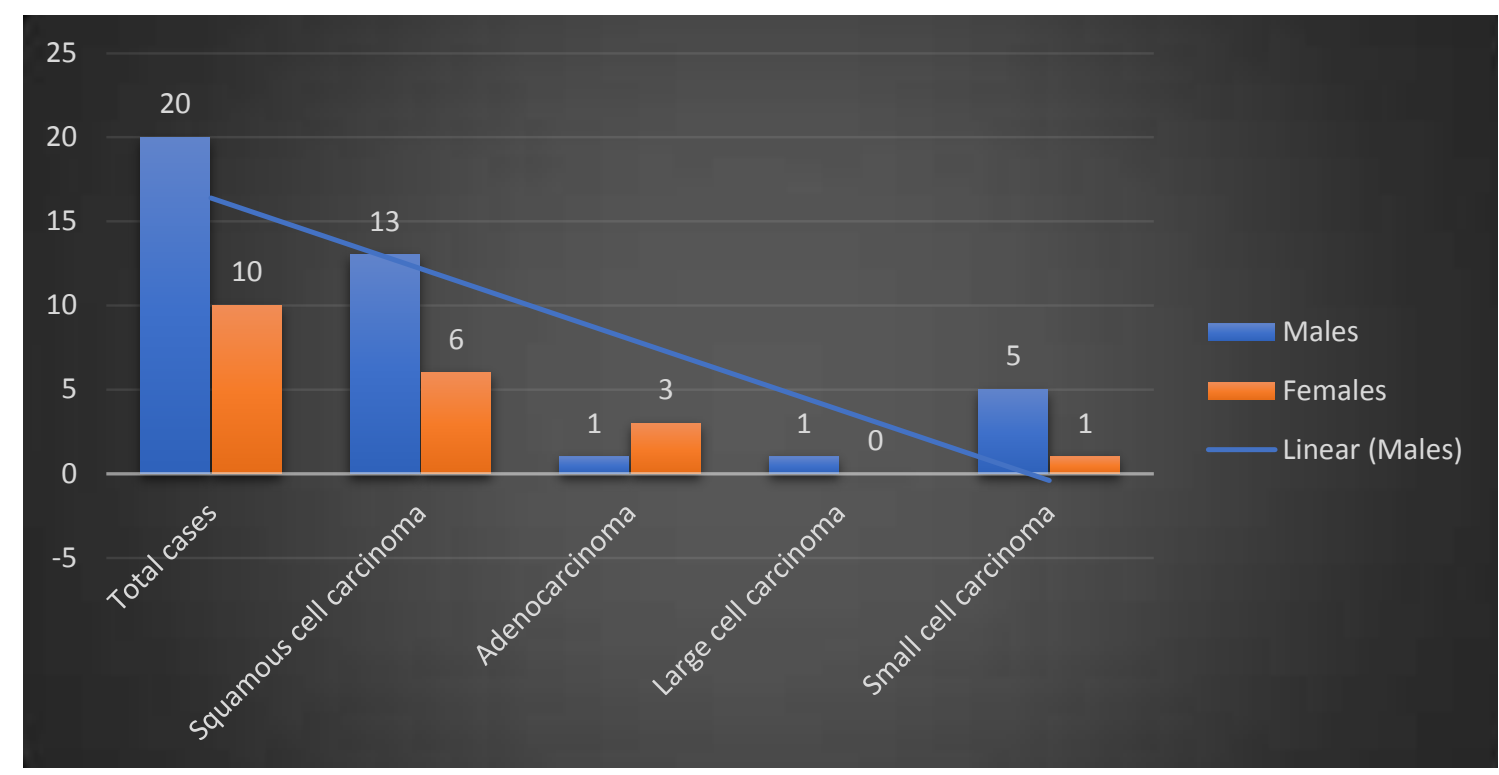

Figure-1:- Frequencies of lung cancer according to types and gender of patients.

Chronic bronchitis was the most frequent type of nonmalignant lung condition constituting 12/30 (40\%), followed by emphysema $6 / 30(20 \%)$, bronchiectasis $5 / 30(16.67 \%)$, lung fibrosis $3 / 30(10 \%)$, pulmonary tuberculosis $2 / 30$ $(6.67 \%)$, and asthma $2 / 30(6.66 \%)$, figure- 2 . 


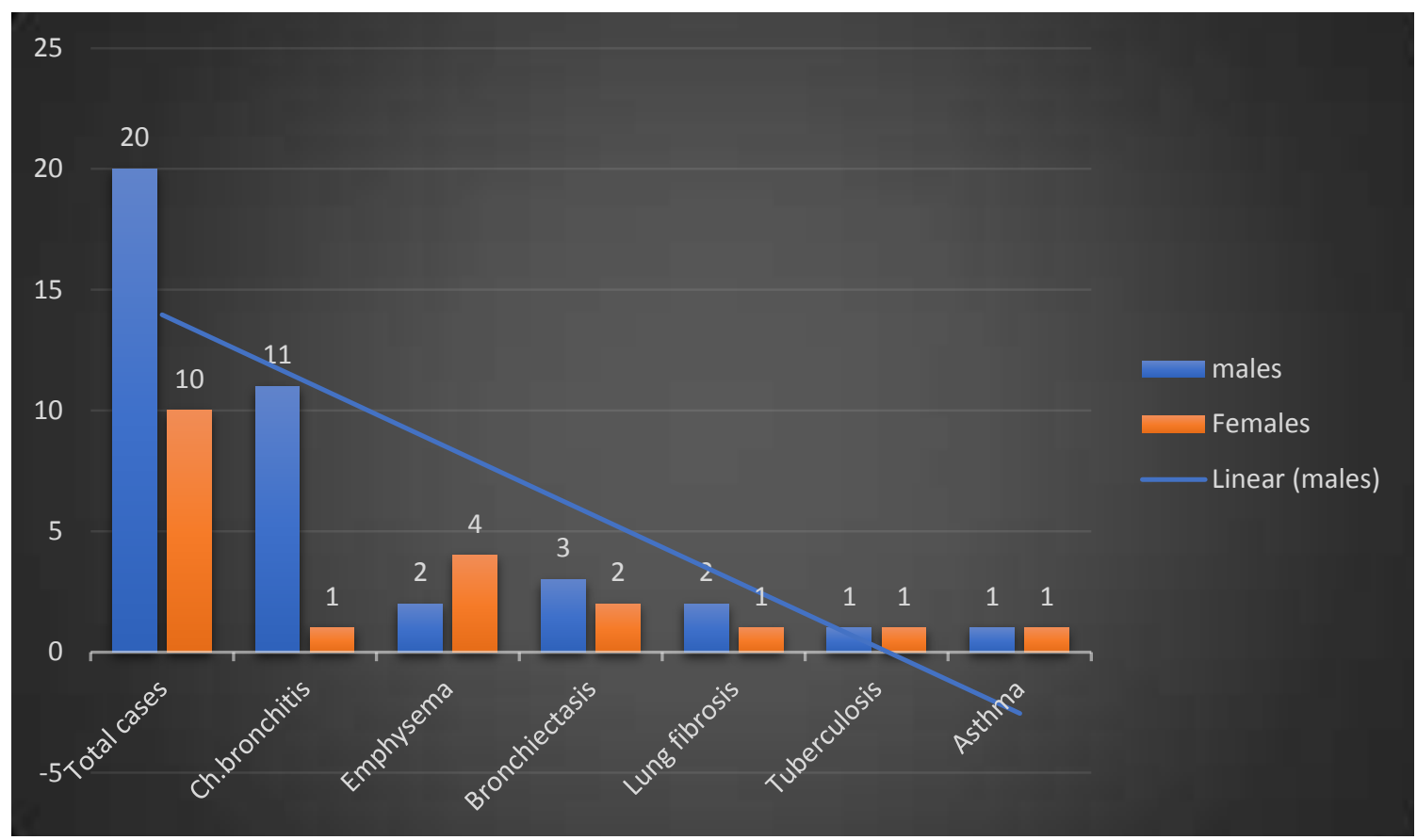

Figure-2:- Frequencies of types of nonmalignant lung conditions and gender distribution.

Table-1:- Expression of studied genes, D scores, and probability of sample being malignant or benign (control) according to D scores in blood samples taken from patients with lung cancer.

\begin{tabular}{|c|c|c|c|c|c|c|c|c|c|c|c|c|c|}
\hline $\begin{array}{l}\text { Diagn } \\
\text { osis }\end{array}$ & $\begin{array}{l}\text { P5 } \\
3\end{array}$ & $\begin{array}{l}\text { KR } \\
\text { AS }\end{array}$ & $\begin{array}{l}\text { cM } \\
\text { YC }\end{array}$ & $\begin{array}{l}\text { He } \\
\text { r- } \\
\text { 2/ } \\
\text { ne } \\
\text { u }\end{array}$ & $\begin{array}{l}\text { Mi } \\
\text { R- } \\
21\end{array}$ & $\begin{array}{l}\text { mi } \\
\text { R- } \\
34 a\end{array}$ & $\begin{array}{l}\text { mi } \\
\text { R- } \\
92\end{array}$ & $\begin{array}{l}\text { mi } \\
\text { R- } \\
\mathbf{9 8}\end{array}$ & $\begin{array}{l}\text { Observe } \\
\text { d group } \\
\text { member } \\
\text { ship }\end{array}$ & $\begin{array}{l}\text { Predicte } \\
\text { d group } \\
\text { member } \\
\text { ship }\end{array}$ & $\begin{array}{l}\text { D- } \\
\text { Sco } \\
\text { re }\end{array}$ & $\begin{array}{l}\text { Probabi } \\
\text { lity of } \\
\text { being } \\
\text { control } \\
(\%)\end{array}$ & $\begin{array}{l}\text { Probabi } \\
\text { lity of } \\
\text { being } \\
\text { Ca }(\%)\end{array}$ \\
\hline $\mathrm{SqCC}$ & $\begin{array}{l}0.2 \\
3\end{array}$ & 0.02 & 0.03 & $\begin{array}{l}0.1 \\
1\end{array}$ & $\begin{array}{l}0.3 \\
9\end{array}$ & $\begin{array}{l}0.0 \\
2\end{array}$ & $\begin{array}{l}0.2 \\
9\end{array}$ & $\begin{array}{l}0.1 \\
6\end{array}$ & Ca cases & Ca cases & 0.1 & 47.5 & 52.5 \\
\hline $\mathrm{SqCC}$ & $\begin{array}{l}0.0 \\
6\end{array}$ & 0.01 & 0.01 & $\begin{array}{l}0.0 \\
1\end{array}$ & $\begin{array}{l}0.1 \\
7\end{array}$ & 0.0 & $\begin{array}{l}0.0 \\
7\end{array}$ & $\begin{array}{l}0.0 \\
4\end{array}$ & Ca cases & Ca cases & 0.2 & 41.2 & 58.8 \\
\hline SqCC & $\begin{array}{l}0.0 \\
3\end{array}$ & 0.31 & 0.13 & $\begin{array}{l}0.0 \\
3\end{array}$ & $\begin{array}{l}0.1 \\
5\end{array}$ & $\begin{array}{l}0.0 \\
2\end{array}$ & $\begin{array}{l}0.0 \\
3\end{array}$ & $\begin{array}{l}0.1 \\
2\end{array}$ & Ca cases & Ca cases & 0.8 & 17.8 & 82.2 \\
\hline $\mathrm{AC}$ & $\begin{array}{l}0.0 \\
1 \\
\end{array}$ & 0.29 & 0.08 & $\begin{array}{l}0.1 \\
1 \\
\end{array}$ & $\begin{array}{l}0.0 \\
1\end{array}$ & 0.0 & $\begin{array}{l}0.3 \\
4\end{array}$ & $\begin{array}{l}0.0 \\
6\end{array}$ & Ca cases & Ca cases & 1.4 & 7.5 & 92.5 \\
\hline $\mathrm{SqCC}$ & $\begin{array}{l}0.0 \\
2 \\
\end{array}$ & 0.02 & 0.03 & $\begin{array}{l}0.0 \\
7 \\
\end{array}$ & $\begin{array}{l}0.2 \\
3 \\
\end{array}$ & $\begin{array}{l}0.0 \\
1 \\
\end{array}$ & $\begin{array}{l}0.2 \\
3 \\
\end{array}$ & $\begin{array}{l}0.1 \\
8 \\
\end{array}$ & Ca cases & Ca cases & 0.2 & 39.2 & 60.8 \\
\hline $\mathrm{SqCC}$ & $\begin{array}{l}0.0 \\
3 \\
\end{array}$ & 0.01 & 0.01 & $\begin{array}{l}0.0 \\
1 \\
\end{array}$ & $\begin{array}{l}0.1 \\
5\end{array}$ & 0.0 & $\begin{array}{l}0.2 \\
1 \\
\end{array}$ & $\begin{array}{l}0.0 \\
1 \\
\end{array}$ & Ca cases & Ca cases & 0.3 & 37.4 & 62.6 \\
\hline SCC & $\begin{array}{l}0.3 \\
8\end{array}$ & 0.02 & 2.0 & $\begin{array}{l}0.6 \\
2\end{array}$ & $\begin{array}{l}0.7 \\
1\end{array}$ & $\begin{array}{l}0.0 \\
1\end{array}$ & $\begin{array}{l}0.0 \\
1\end{array}$ & $\begin{array}{l}2.6 \\
4\end{array}$ & Ca cases & Ca cases & 1.1 & 12.3 & 87.7 \\
\hline SCC & $\begin{array}{l}0.0 \\
3 \\
\end{array}$ & 0.03 & 2.0 & 0.5 & $\begin{array}{l}0.7 \\
7 \\
\end{array}$ & $\begin{array}{l}0.0 \\
1 \\
\end{array}$ & $\begin{array}{l}0.1 \\
3 \\
\end{array}$ & 6.5 & Ca cases & Ca cases & 2.7 & 0.7 & 99.3 \\
\hline SqCC & $\begin{array}{l}0.0 \\
3\end{array}$ & 0.13 & 0.05 & $\begin{array}{l}0.3 \\
8\end{array}$ & $\begin{array}{l}0.3 \\
3\end{array}$ & $\begin{array}{l}0.0 \\
2\end{array}$ & $\begin{array}{l}0.1 \\
4\end{array}$ & $\begin{array}{l}0.1 \\
3\end{array}$ & Ca cases & Ca cases & 0.6 & 24.0 & 76.0 \\
\hline $\mathrm{SqCC}$ & $\begin{array}{l}0.0 \\
1\end{array}$ & 0.01 & 0.01 & $\begin{array}{l}0.0 \\
1\end{array}$ & $\begin{array}{l}0.1 \\
4\end{array}$ & $\begin{array}{l}0.0 \\
1\end{array}$ & 0.2 & $\begin{array}{l}0.0 \\
1\end{array}$ & Ca cases & Ca cases & 0.0 & 48.4 & 51.6 \\
\hline SqCC & $\begin{array}{l}0.0 \\
3\end{array}$ & 0.02 & 0.02 & $\begin{array}{l}0.0 \\
2\end{array}$ & $\begin{array}{l}0.0 \\
2\end{array}$ & $\begin{array}{l}0.0 \\
2\end{array}$ & $\begin{array}{l}0.2 \\
5\end{array}$ & $\begin{array}{l}0.0 \\
7\end{array}$ & Ca cases & $\begin{array}{l}\text { Benign } \\
\text { cases }\end{array}$ & -0.2 & 57.2 & 42.8 \\
\hline $\mathrm{SqCC}$ & $\begin{array}{l}0.0 \\
1 \\
\end{array}$ & 0.01 & 0.07 & $\begin{array}{l}0.0 \\
1 \\
\end{array}$ & $\begin{array}{l}0.2 \\
9\end{array}$ & 0.0 & $\begin{array}{l}0.2 \\
1 \\
\end{array}$ & $\begin{array}{l}0.0 \\
5\end{array}$ & Ca cases & Ca cases & 0.3 & 35.1 & 64.9 \\
\hline
\end{tabular}




\begin{tabular}{|c|c|c|c|c|c|c|c|c|c|c|c|c|c|}
\hline $\mathrm{SqCC}$ & $\begin{array}{l}0.0 \\
4\end{array}$ & 0.04 & 0.04 & $\begin{array}{l}0.0 \\
4\end{array}$ & $\begin{array}{l}0.3 \\
7\end{array}$ & $\begin{array}{l}0.0 \\
1\end{array}$ & $\begin{array}{l}0.5 \\
5\end{array}$ & $\begin{array}{l}0.0 \\
8\end{array}$ & Ca cases & Ca cases & 0.5 & 28.9 & 71.1 \\
\hline $\mathrm{SqCC}$ & $\begin{array}{l}0.2 \\
1\end{array}$ & 0.02 & 0.04 & $\begin{array}{l}0.0 \\
3\end{array}$ & $\begin{array}{l}0.6 \\
9\end{array}$ & $\begin{array}{l}0.0 \\
1\end{array}$ & $\begin{array}{l}0.1 \\
1\end{array}$ & $\begin{array}{l}0.0 \\
1\end{array}$ & Ca cases & Ca cases & 0.1 & 43.5 & 56.5 \\
\hline SqCC & $\begin{array}{l}0.2 \\
3 \\
\end{array}$ & 0.01 & 0.15 & $\begin{array}{l}0.0 \\
2 \\
\end{array}$ & $\begin{array}{l}0.2 \\
3 \\
\end{array}$ & 0.0 & $\begin{array}{l}0.0 \\
2 \\
\end{array}$ & $\begin{array}{l}0.1 \\
1 \\
\end{array}$ & Ca cases & Ca cases & 0.1 & 44.6 & 55.4 \\
\hline $\mathrm{SqCC}$ & $\begin{array}{l}0.0 \\
5 \\
\end{array}$ & 0.11 & 0.06 & $\begin{array}{l}0.0 \\
6 \\
\end{array}$ & $\begin{array}{l}1.6 \\
2\end{array}$ & $\begin{array}{l}0.0 \\
1 \\
\end{array}$ & $\begin{array}{l}0.4 \\
4 \\
\end{array}$ & $\begin{array}{l}0.1 \\
2 \\
\end{array}$ & Ca cases & Ca cases & 1.1 & 11.5 & 88.5 \\
\hline SqCC & $\begin{array}{l}0.3 \\
1 \\
\end{array}$ & 0.11 & 0.11 & $\begin{array}{l}0.0 \\
8 \\
\end{array}$ & $\begin{array}{l}0.8 \\
5 \\
\end{array}$ & $\begin{array}{l}0.0 \\
3 \\
\end{array}$ & $\begin{array}{l}4.6 \\
3 \\
\end{array}$ & 0.1 & Ca cases & Ca cases & 3.1 & 0.3 & 99.7 \\
\hline LCC & $\begin{array}{l}0.0 \\
1 \\
\end{array}$ & 0.01 & 0.09 & 0.2 & $\begin{array}{l}0.1 \\
9\end{array}$ & $\begin{array}{l}0.0 \\
1 \\
\end{array}$ & $\begin{array}{l}0.0 \\
7 \\
\end{array}$ & $\begin{array}{l}0.0 \\
2 \\
\end{array}$ & Ca cases & Ca cases & 0.1 & 43.6 & 56.4 \\
\hline $\mathrm{SqCC}$ & $\begin{array}{l}0.0 \\
4 \\
\end{array}$ & 0.08 & 0.04 & $\begin{array}{l}0.0 \\
3 \\
\end{array}$ & $\begin{array}{l}0.1 \\
5 \\
\end{array}$ & 0.0 & $\begin{array}{l}0.3 \\
1 \\
\end{array}$ & $\begin{array}{l}0.0 \\
2 \\
\end{array}$ & Ca cases & Ca cases & 0.6 & 25.1 & 74.9 \\
\hline $\mathrm{SqCC}$ & $\begin{array}{l}0.0 \\
6 \\
\end{array}$ & 0.05 & 0.09 & $\begin{array}{l}0.0 \\
6 \\
\end{array}$ & $\begin{array}{l}0.6 \\
9 \\
\end{array}$ & $\begin{array}{l}0.0 \\
3 \\
\end{array}$ & $\begin{array}{l}0.3 \\
2 \\
\end{array}$ & $\begin{array}{l}0.3 \\
5 \\
\end{array}$ & Ca cases & Ca cases & 0.1 & 45.4 & 54.6 \\
\hline SCC & $\begin{array}{l}0.0 \\
2 \\
\end{array}$ & 0.01 & 3.53 & $\begin{array}{l}0.2 \\
7 \\
\end{array}$ & $\begin{array}{l}0.0 \\
4 \\
\end{array}$ & $\begin{array}{l}0.0 \\
2 \\
\end{array}$ & $\begin{array}{l}0.0 \\
4 \\
\end{array}$ & $\begin{array}{l}4.9 \\
6 \\
\end{array}$ & Ca cases & Ca cases & 0.8 & 18.7 & 81.3 \\
\hline $\mathrm{SqCC}$ & $\begin{array}{l}0.0 \\
2 \\
\end{array}$ & 0.02 & 0.04 & $\begin{array}{l}0.0 \\
2 \\
\end{array}$ & 0.1 & 0.0 & $\begin{array}{l}0.2 \\
5 \\
\end{array}$ & $\begin{array}{l}0.0 \\
1 \\
\end{array}$ & Ca cases & Ca cases & 0.3 & 35.4 & 64.6 \\
\hline SCC & $\begin{array}{l}0.1 \\
3 \\
\end{array}$ & 0.04 & 3.66 & $\begin{array}{l}0.0 \\
6 \\
\end{array}$ & $\begin{array}{l}5.3 \\
5 \\
\end{array}$ & $\begin{array}{l}0.0 \\
2 \\
\end{array}$ & $\begin{array}{l}0.0 \\
3 \\
\end{array}$ & $\begin{array}{l}6.5 \\
4 \\
\end{array}$ & Ca cases & Ca cases & 3.0 & 0.4 & 99.6 \\
\hline $\mathrm{SqCC}$ & $\begin{array}{l}0.0 \\
5 \\
\end{array}$ & 0.14 & 0.07 & $\begin{array}{l}0.0 \\
7 \\
\end{array}$ & $\begin{array}{l}0.4 \\
1 \\
\end{array}$ & $\begin{array}{l}0.0 \\
1 \\
\end{array}$ & $\begin{array}{l}0.0 \\
6 \\
\end{array}$ & $\begin{array}{l}0.0 \\
4 \\
\end{array}$ & Ca cases & Ca cases & 0.5 & 28.1 & 71.9 \\
\hline SCC & $\begin{array}{l}0.0 \\
3 \\
\end{array}$ & 0.01 & 2.63 & $\begin{array}{l}0.0 \\
3 \\
\end{array}$ & $\begin{array}{l}0.0 \\
2 \\
\end{array}$ & $\begin{array}{l}0.0 \\
1 \\
\end{array}$ & $\begin{array}{l}0.0 \\
2 \\
\end{array}$ & $\begin{array}{l}3.8 \\
9 \\
\end{array}$ & Ca cases & Ca cases & 0.7 & 21.6 & 78.4 \\
\hline $\mathrm{AC}$ & $\begin{array}{l}1.5 \\
9 \\
\end{array}$ & 0.47 & 1.01 & $\begin{array}{l}1.4 \\
2 \\
\end{array}$ & $\begin{array}{l}1.5 \\
8 \\
\end{array}$ & $\begin{array}{l}0.0 \\
3 \\
\end{array}$ & 1.3 & $\begin{array}{l}0.4 \\
5 \\
\end{array}$ & Ca cases & Ca cases & 3.1 & 0.3 & 99.7 \\
\hline SCC & $\begin{array}{l}0.0 \\
8\end{array}$ & 0.5 & 7.67 & $\begin{array}{l}0.2 \\
5 \\
\end{array}$ & $\begin{array}{l}1.0 \\
6 \\
\end{array}$ & $\begin{array}{l}0.0 \\
2\end{array}$ & $\begin{array}{l}0.0 \\
7 \\
\end{array}$ & $\begin{array}{l}7.1 \\
1\end{array}$ & Ca cases & Ca cases & 2.4 & 1.2 & 98.8 \\
\hline $\mathrm{AC}$ & $\begin{array}{l}1.1 \\
8\end{array}$ & 0.07 & 0.07 & $\begin{array}{l}2.5 \\
5 \\
\end{array}$ & $\begin{array}{l}0.2 \\
4 \\
\end{array}$ & $\begin{array}{l}0.0 \\
3 \\
\end{array}$ & $\begin{array}{l}0.0 \\
3 \\
\end{array}$ & $\begin{array}{l}0.3 \\
8 \\
\end{array}$ & Ca cases & Ca cases & 2.0 & 2.5 & 97.5 \\
\hline $\mathrm{SqCC}$ & $\begin{array}{l}0.0 \\
3 \\
\end{array}$ & 0.24 & 0.04 & $\begin{array}{l}0.0 \\
4 \\
\end{array}$ & $\begin{array}{l}0.0 \\
3 \\
\end{array}$ & $\begin{array}{l}0.0 \\
4 \\
\end{array}$ & $\begin{array}{l}1.2 \\
9 \\
\end{array}$ & $\begin{array}{l}0.0 \\
2 \\
\end{array}$ & Ca cases & Ca cases & 0.8 & 18.8 & 81.2 \\
\hline $\mathrm{AC}$ & 1.0 & 0.23 & 0.42 & $\begin{array}{l}0.2 \\
5 \\
\end{array}$ & $\begin{array}{l}0.3 \\
7\end{array}$ & $\begin{array}{l}0.0 \\
1 \\
\end{array}$ & $\begin{array}{l}0.4 \\
7\end{array}$ & $\begin{array}{l}0.2 \\
5\end{array}$ & Ca cases & Ca cases & 0.9 & 15.8 & 84.2 \\
\hline
\end{tabular}

Table-2:- Expression of genes, D scores, and probability of sample being malignant or benign (control) according to D scores in blood samples taken from patients with benign lung conditions.

\begin{tabular}{|c|c|c|c|c|c|c|c|c|c|c|c|c|c|}
\hline $\begin{array}{l}\text { Diag } \\
\text { n- } \\
\text { osis }\end{array}$ & P53 & $\begin{array}{l}\text { KR } \\
\text { AS }\end{array}$ & $\begin{array}{l}\text { cM } \\
\text { YC }\end{array}$ & $\begin{array}{l}\text { He } \\
\text { r- } \\
2 \text { ne } \\
\text { u }\end{array}$ & $\begin{array}{l}\text { mi } \\
\text { R- } \\
21\end{array}$ & $\begin{array}{l}\text { mi } \\
\text { R- } \\
\text { 34a }\end{array}$ & $\begin{array}{l}\text { mi } \\
\text { R- } \\
92\end{array}$ & $\begin{array}{l}\text { mi } \\
\text { R- } \\
98\end{array}$ & $\begin{array}{l}\text { Observe } \\
\text { d Group } \\
\text { members } \\
\text { hip }\end{array}$ & $\begin{array}{l}\text { Predicte } \\
\text { d group } \\
\text { members } \\
\text { hip }\end{array}$ & $\begin{array}{l}\text { D - } \\
\text { sco } \\
\text { re }\end{array}$ & $\begin{array}{l}\text { Probabi } \\
\text { lity of } \\
\text { being } \\
\text { control } \\
(\%)\end{array}$ & $\begin{array}{l}\text { Probabi } \\
\text { lity of } \\
\text { being } \\
\text { Ca }(\%)\end{array}$ \\
\hline $\begin{array}{l}\text { Infla } \\
\text { m }\end{array}$ & $\begin{array}{l}0.0 \\
2 \\
\end{array}$ & 0.01 & 0.01 & $\begin{array}{l}0.0 \\
2 \\
\end{array}$ & $\begin{array}{l}0.0 \\
4 \\
\end{array}$ & $\begin{array}{l}0.0 \\
3 \\
\end{array}$ & $\begin{array}{l}0.0 \\
6 \\
\end{array}$ & $\begin{array}{l}0.0 \\
1 \\
\end{array}$ & Controls & Controls & -0.6 & 74.1 & 25.9 \\
\hline $\begin{array}{l}\text { Infla } \\
\text { m }\end{array}$ & $\begin{array}{l}0.1 \\
2\end{array}$ & 0.03 & 0.19 & $\begin{array}{l}0.0 \\
4\end{array}$ & $\begin{array}{l}0.0 \\
9\end{array}$ & $\begin{array}{l}0.0 \\
4\end{array}$ & $\begin{array}{l}0.2 \\
9\end{array}$ & $\begin{array}{l}0.2 \\
6\end{array}$ & Controls & Controls & -0.5 & 73.1 & 26.9 \\
\hline $\begin{array}{l}\text { Infla } \\
\text { m }\end{array}$ & $\begin{array}{l}0.0 \\
1\end{array}$ & 0.04 & 0.05 & $\begin{array}{l}0.0 \\
2\end{array}$ & $\begin{array}{l}0.0 \\
2\end{array}$ & $\begin{array}{l}0.0 \\
3\end{array}$ & $\begin{array}{l}0.0 \\
2\end{array}$ & $\begin{array}{l}0.0 \\
5\end{array}$ & Controls & Controls & -0.5 & 71.4 & 28.6 \\
\hline $\begin{array}{l}\text { Infla } \\
\text { m }\end{array}$ & $\begin{array}{l}0.0 \\
2\end{array}$ & 0.01 & 0.02 & 0.0 & $\begin{array}{l}0.0 \\
1\end{array}$ & $\begin{array}{l}0.0 \\
1\end{array}$ & $\begin{array}{l}0.0 \\
3\end{array}$ & $\begin{array}{l}0.0 \\
1\end{array}$ & Controls & Controls & -0.1 & 56.5 & 37.4 \\
\hline $\begin{array}{l}\text { Infla } \\
\text { m }\end{array}$ & $\begin{array}{l}0.0 \\
2\end{array}$ & 0.03 & 0.01 & $\begin{array}{l}0.0 \\
4\end{array}$ & $\begin{array}{l}0.0 \\
1\end{array}$ & $\begin{array}{l}0.0 \\
2\end{array}$ & $\begin{array}{l}0.0 \\
2\end{array}$ & $\begin{array}{l}0.0 \\
1\end{array}$ & Controls & Controls & -0.3 & 62.6 & 37.4 \\
\hline Infla & 0.1 & 0.04 & 0.03 & 0.0 & 0.1 & 0.1 & 0.0 & 0.0 & Controls & Controls & -3.3 & 99.8 & 0.20 \\
\hline
\end{tabular}




\begin{tabular}{|c|c|c|c|c|c|c|c|c|c|c|c|c|c|}
\hline $\mathrm{m}$ & 4 & & & 8 & & 5 & 4 & 8 & & & & & \\
\hline $\begin{array}{l}\text { Infla } \\
\text { m }\end{array}$ & $\begin{array}{l}0.3 \\
1\end{array}$ & \begin{tabular}{|l|l|}
0.16 \\
\end{tabular} & 0.07 & $\begin{array}{l}\text { 0.0 } \\
6\end{array}$ & $\begin{array}{l}0.0 \\
3\end{array}$ & $\begin{array}{l}0.0 \\
2\end{array}$ & $\begin{array}{l}\text { 0.0 } \\
2\end{array}$ & $\begin{array}{l}\text { 0.0 } \\
6\end{array}$ & Controls & Ca cases & 0.1 & 46.1 & 53.9 \\
\hline $\begin{array}{l}\text { Infla } \\
\mathrm{m}\end{array}$ & $\begin{array}{l}0.0 \\
2\end{array}$ & 0.01 & 0.04 & $\begin{array}{l}0.0 \\
2\end{array}$ & $\begin{array}{l}0.0 \\
6\end{array}$ & $\begin{array}{l}0.0 \\
8\end{array}$ & $\begin{array}{l}0.0 \\
6\end{array}$ & $\begin{array}{l}0.0 \\
5\end{array}$ & Controls & Controls & -1.8 & 96.3 & 3.7 \\
\hline $\begin{array}{l}\text { Infla } \\
\mathrm{m}\end{array}$ & $\begin{array}{l}0.0 \\
1\end{array}$ & 0.03 & 0.01 & $\begin{array}{l}0.0 \\
2\end{array}$ & $\begin{array}{l}0.0 \\
1\end{array}$ & $\begin{array}{l}0.0 \\
1\end{array}$ & $\begin{array}{l}0.0 \\
2\end{array}$ & $\begin{array}{l}0.0 \\
4\end{array}$ & Controls & Controls & 0.0 & 52 & 48 \\
\hline $\begin{array}{l}\text { Infla } \\
\mathrm{m}\end{array}$ & $\begin{array}{l}0.0 \\
3 \\
\end{array}$ & 0.14 & 0.07 & $\begin{array}{l}0.0 \\
6 \\
\end{array}$ & $\begin{array}{l}0.0 \\
7 \\
\end{array}$ & $\begin{array}{l}0.1 \\
9 \\
\end{array}$ & $\begin{array}{l}0.1 \\
4 \\
\end{array}$ & $\begin{array}{l}0.0 \\
7 \\
\end{array}$ & Controls & Controls & -3.9 & 99.9 & 0.1 \\
\hline $\begin{array}{l}\text { Infla } \\
\text { m }\end{array}$ & 0.0 & 0.01 & 0.01 & $\begin{array}{l}0.0 \\
1\end{array}$ & 0.0 & 0.0 & 0.0 & 0.0 & Controls & Ca cases & 0.1 & 45.7 & 54.3 \\
\hline $\begin{array}{l}\text { Infla } \\
\text { m }\end{array}$ & $\begin{array}{l}0.0 \\
4\end{array}$ & 0.02 & 0.08 & $\begin{array}{l}0.0 \\
4\end{array}$ & $\begin{array}{l}0.0 \\
1\end{array}$ & $\begin{array}{l}0.0 \\
4\end{array}$ & $\begin{array}{l}0.0 \\
4\end{array}$ & $\begin{array}{l}0.0 \\
2\end{array}$ & Controls & Controls & -0.8 & 80.6 & 19.4 \\
\hline $\begin{array}{l}\text { Infla } \\
\text { m }\end{array}$ & $\begin{array}{l}0.0 \\
1\end{array}$ & 0.02 & 0.01 & $\begin{array}{l}0.0 \\
4\end{array}$ & $\begin{array}{l}0.0 \\
1\end{array}$ & $\begin{array}{l}0.0 \\
5\end{array}$ & $\begin{array}{l}0.0 \\
2\end{array}$ & $\begin{array}{l}0.0 \\
3\end{array}$ & Controls & Controls & -1.0 & 86.9 & 13.1 \\
\hline $\begin{array}{l}\text { Infla } \\
\text { m }\end{array}$ & $\begin{array}{l}0.0 \\
9\end{array}$ & 0.03 & 0.14 & $\begin{array}{l}0.0 \\
5\end{array}$ & $\begin{array}{l}0.0 \\
8\end{array}$ & $\begin{array}{l}0.0 \\
5\end{array}$ & $\begin{array}{l}0.1 \\
6\end{array}$ & $\begin{array}{l}0.0 \\
4\end{array}$ & Controls & Controls & -0.9 & 84.6 & 15.4 \\
\hline $\begin{array}{l}\text { Infla } \\
\text { m }\end{array}$ & $\begin{array}{l}0.0 \\
2\end{array}$ & 0.04 & 0.02 & $\begin{array}{l}0.0 \\
2\end{array}$ & $\begin{array}{l}0.0 \\
3\end{array}$ & $\begin{array}{l}0.0 \\
2\end{array}$ & $\begin{array}{l}0.0 \\
4\end{array}$ & $\begin{array}{l}0.0 \\
1\end{array}$ & Controls & Controls & -0.2 & 61.2 & 38.8 \\
\hline $\begin{array}{l}\text { Infla } \\
\mathrm{m}\end{array}$ & $\begin{array}{l}0.0 \\
1\end{array}$ & 0.01 & 0.03 & $\begin{array}{l}0.0 \\
1\end{array}$ & $\begin{array}{l}0.0 \\
1\end{array}$ & $\begin{array}{l}0.0 \\
2\end{array}$ & $\begin{array}{l}0.0 \\
1\end{array}$ & $\begin{array}{l}0.0 \\
1\end{array}$ & Controls & Controls & -0.4 & 67 & 33 \\
\hline $\begin{array}{l}\text { Infla } \\
\mathrm{m}\end{array}$ & $\begin{array}{l}0.0 \\
3 \\
\end{array}$ & 0.11 & 0.15 & $\begin{array}{l}0.0 \\
8 \\
\end{array}$ & $\begin{array}{l}0.5 \\
3 \\
\end{array}$ & $\begin{array}{l}0.0 \\
5 \\
\end{array}$ & $\begin{array}{l}0.1 \\
1\end{array}$ & $\begin{array}{l}0.0 \\
4\end{array}$ & Controls & Controls & -0.5 & 71.1 & 28.9 \\
\hline $\begin{array}{l}\text { Infla } \\
\mathrm{m}\end{array}$ & $\begin{array}{l}0.0 \\
1\end{array}$ & 0.0 & 0.03 & $\begin{array}{l}0.0 \\
2\end{array}$ & $\begin{array}{l}0.0 \\
2\end{array}$ & $\begin{array}{l}0.0 \\
1\end{array}$ & $\begin{array}{l}0.0 \\
3\end{array}$ & $\begin{array}{l}0.0 \\
1\end{array}$ & Controls & Controls & -0.1 & 56.9 & 43.1 \\
\hline $\begin{array}{l}\text { Infla } \\
\text { m }\end{array}$ & $\begin{array}{l}0.0 \\
1\end{array}$ & 0.06 & 0.03 & $\begin{array}{l}0.0 \\
3\end{array}$ & $\begin{array}{l}0.0 \\
3\end{array}$ & $\begin{array}{l}0.0 \\
3\end{array}$ & $\begin{array}{l}0.0 \\
9\end{array}$ & $\begin{array}{l}0.0 \\
7\end{array}$ & Controls & Controls & -0.4 & 65.8 & 34.2 \\
\hline $\begin{array}{l}\text { Infla } \\
\text { m }\end{array}$ & $\begin{array}{l}0.0 \\
12\end{array}$ & 0.01 & 0.03 & $\begin{array}{l}0.0 \\
1\end{array}$ & $\begin{array}{l}0.0 \\
1\end{array}$ & $\begin{array}{l}0.0 \\
1\end{array}$ & $\begin{array}{l}0.0 \\
4\end{array}$ & $\begin{array}{l}0.0 \\
1\end{array}$ & Controls & Controls & -0.1 & 55.7 & 44.3 \\
\hline $\begin{array}{l}\text { Infla } \\
\mathrm{m}\end{array}$ & $\begin{array}{l}0.0 \\
4\end{array}$ & 0.05 & 0.09 & $\begin{array}{l}0.0 \\
2\end{array}$ & $\begin{array}{l}0.0 \\
3\end{array}$ & $\begin{array}{l}0.0 \\
9\end{array}$ & $\begin{array}{l}0.0 \\
4\end{array}$ & $\begin{array}{l}0.0 \\
4\end{array}$ & Controls & Controls & -1.9 & 97.2 & 2.8 \\
\hline $\begin{array}{l}\text { Infla } \\
\text { m }\end{array}$ & $\begin{array}{l}0.0 \\
2\end{array}$ & 0.15 & 0.11 & $\begin{array}{l}0.1 \\
3\end{array}$ & $\begin{array}{l}0.0 \\
4\end{array}$ & $\begin{array}{l}0.0 \\
4\end{array}$ & $\begin{array}{l}0.0 \\
8\end{array}$ & $\begin{array}{l}0.0 \\
3\end{array}$ & Controls & Controls & -0.2 & 60.9 & 39.1 \\
\hline $\begin{array}{l}\text { Infla } \\
\text { m }\end{array}$ & $\begin{array}{l}0.0 \\
2\end{array}$ & 0.02 & 0.04 & $\begin{array}{l}0.0 \\
1\end{array}$ & $\begin{array}{l}0.0 \\
5\end{array}$ & $\begin{array}{l}0.0 \\
4\end{array}$ & $\begin{array}{l}0.0 \\
2\end{array}$ & $\begin{array}{l}0.0 \\
1\end{array}$ & Controls & Controls & -0.8 & 81.9 & 18.1 \\
\hline $\begin{array}{l}\text { Infla } \\
\mathrm{m}\end{array}$ & $\begin{array}{l}0.0 \\
6\end{array}$ & 0.07 & 0.13 & $\begin{array}{l}0.0 \\
7\end{array}$ & $\begin{array}{l}0.0 \\
6 \\
\end{array}$ & $\begin{array}{l}0.1 \\
3\end{array}$ & $\begin{array}{l}0.0 \\
4\end{array}$ & 0.1 & Controls & Controls & -2.7 & 99.4 & 0.6 \\
\hline $\begin{array}{l}\text { Infla } \\
\text { m }\end{array}$ & $\begin{array}{l}0.0 \\
2\end{array}$ & 0.02 & 0.04 & $\begin{array}{l}0.0 \\
1\end{array}$ & $\begin{array}{l}0.0 \\
8\end{array}$ & $\begin{array}{l}0.0 \\
6\end{array}$ & $\begin{array}{l}0.0 \\
4\end{array}$ & $\begin{array}{l}0.0 \\
2\end{array}$ & Controls & Controls & -1.3 & 91.3 & 8.7 \\
\hline $\begin{array}{l}\text { Infla } \\
\mathrm{m}\end{array}$ & $\begin{array}{l}0.0 \\
1\end{array}$ & 0.01 & 0.02 & $\begin{array}{l}0.0 \\
1\end{array}$ & $\begin{array}{l}0.0 \\
1\end{array}$ & $\begin{array}{l}0.0 \\
2\end{array}$ & $\begin{array}{l}0.0 \\
12\end{array}$ & $\begin{array}{l}0.0 \\
1\end{array}$ & Controls & Controls & -0.4 & 66.9 & 33.1 \\
\hline $\begin{array}{l}\text { Infla } \\
\text { m }\end{array}$ & $\begin{array}{l}0.4 \\
2\end{array}$ & 0.06 & 0.21 & $\begin{array}{l}0.1 \\
1\end{array}$ & $\begin{array}{l}0.0 \\
6\end{array}$ & $\begin{array}{l}0.1 \\
1\end{array}$ & $\begin{array}{l}0.0 \\
4\end{array}$ & $\begin{array}{l}0.0 \\
7\end{array}$ & Controls & Controls & -2.4 & 98.9 & 1.1 \\
\hline $\begin{array}{l}\text { Infla } \\
\mathrm{m}\end{array}$ & 027 & 0.18 & 0.06 & $\begin{array}{l}0.1 \\
4\end{array}$ & $\begin{array}{l}0.0 \\
6\end{array}$ & $\begin{array}{l}0.0 \\
7\end{array}$ & $\begin{array}{l}0.2 \\
2\end{array}$ & $\begin{array}{l}0.0 \\
4\end{array}$ & Controls & Controls & -0.8 & 81.9 & 18.1 \\
\hline $\begin{array}{l}\text { Infla } \\
\text { m }\end{array}$ & $\begin{array}{l}0.0 \\
1\end{array}$ & 0.02 & 0.07 & $\begin{array}{l}0.0 \\
2\end{array}$ & $\begin{array}{l}0.0 \\
2\end{array}$ & $\begin{array}{l}0.0 \\
3\end{array}$ & $\begin{array}{l}0.0 \\
1\end{array}$ & $\begin{array}{l}0.0 \\
2\end{array}$ & Controls & Controls & -0.6 & 74.7 & 25.3 \\
\hline $\begin{array}{l}\text { Infla } \\
\text { m }\end{array}$ & $\begin{array}{l}0.0 \\
3\end{array}$ & \begin{tabular}{|l|}
0.07 \\
\end{tabular} & 0.02 & $\begin{array}{l}0.0 \\
3\end{array}$ & $\begin{array}{l}0.0 \\
3\end{array}$ & $\begin{array}{l}0.0 \\
7\end{array}$ & $\begin{array}{l}0.0 \\
4\end{array}$ & $\begin{array}{l}0.0 \\
3\end{array}$ & Controls & Controls & -1.3 & 92.2 & 7.8 \\
\hline
\end{tabular}

Discussion:-

Not surprisingly, although with a little bit difference from other studies, the highest frequency of patients with lung cancer participated in this study was in the age group of $60-69$ with a mean age of 61.8 years. In similar studies, the mean age of patients with lung cancer was found to be 55.2 by Al-Alusi [32], 54.2 by Menkhi [33], 54.4 by Saeed [34], 57.3 by Thabit [35], 54.4 by Kersting [36], and by Abdul-Majeed [37]. Males constituted 80\% and 
females $20 \%$ of patients in the present study, while males constituted $82.76 \%$ by AL-Alusi [32], 84.62\% by Menkhi [33], 83.6\% by Saeed [34], 83.6 \% by Thabit [35], 84.84 \% by Kersting [36], and 83.3 \% by Abdul-Majeed [37]. Squamous cell carcinoma constituted the highest frequency of lung cancer in present study diagnosed by cytolopathology of bronchial wash and/or histopathology on tissue specimens forming $63.33 \%$, while it only constituted $42 \%$ by AL- Alusi [32], $43.3 \%$ by Menkhi [33], 61\% by Saeed [34], 40\% by Thabit [35], and 43\% by Kersting [36]. The minor differences observed in the present study regarding the above parameters are probably due to the type of the samples included in these studies. Many of these studies depended on the histological diagnosis of tissue sections made from resected lung specimens or biopsies. Cytopathological study of bronchial samples, bronchial washes, brushes, and biopsies are considered of high sensitivity in detection of lung cancer and its types. It can give an accurate diagnosis but subjecting the patients to invasive surgical procedures, this is true when realizing that most of the patients are old ages and have many chronic medical illnesses. Method of discrimination score is an easy completely noninvasive procedure accomplished on venous blood samples. Until now and up to present knowledge, studies combining all the 8 genes employed in the present work are not present. This makes the present study a novel one. For this reason, the collected data of the present study were used to make a discriminant score. This score is going to suit any new blood sample subjected in the same gene expression analysis. So that, there will be no need to do all the analysis presented in this work. Each new blood sample with D score $>0.0$ is going to be considered malignant while sample with $\mathrm{D}$ score of $<0.0$ is going to be considered benign. The higher the positive value of $\mathrm{D}$ score the more confident the tested specimen is really a cancer case. The lower the negative value of $\mathrm{D}$ score the more confident the tested specimen is not a cancer case. The discriminant models used provided an overall accuracy of $95 \%$ when it was applied on each sample included in present study. The discrimination score is employed in many fields such as performance of students in schools or colleges, performance of large companies and banks. In medicine, it was used evaluating response to treatment but no study has employed D score in diagnosis of malignant benign conditions. Paone [38], presented a model of discriminant analysis on SCLC and NSCLC of using a panel of serum markers. These included neurone specific enolase (NSE), cytokeratin fragment antigen- 21.1, CyFRA-21.1, tissue poly-peptide antigen and carcinoembryonic antigen. The markers were studied in sera of 50 NSCLC and 17SCLC. The variables selected were NSE and CyFRA-21.1 considered together, they gave 97\% rate of correct classification. The formula was tested on a group of 7 SCLC and 22 SNCLC patients giving only 2 errors. Marchevsky [39] measured DNA methylation levels at 20 loci in 41 SCLC and 46 NSCLC cell lines with the quantitative realtime PCR method methyl light. The data were analyzed with artificial neural networks (ANN) and linear discriminant analysis (LDA) to classify the cell lines into NSCLC or SCLC. They reached an accuracy of $87 \%$ to $100 \%$ in ANN and $62.5 \%$ to $87.5 \%$ in LDA. Paone [38] evaluated the response to chemotherapy in patients affected with NSCLC by means of three tumor markers elaborated by discriminant analysis. These markers were tissue polypeptide antigen (TPA), CyFRA-21.1, NSE, and carcinoembryonic antigen (CEA). Serum levels of these markers were measured before and after chemotherapy and after 3 cycles of treatment. Discriminant analysis could correctly classify $87.8 \%$ of 74 subjects including $(86.1 \%$ of 36 progressive diseases and $89.5 \%$ of 38 nonprogressive diseases).

\section{Conclusion:-}

According to results from present study any new case of lung condition with a Discriminant Score of $>0.0$ is considered as a lung cancer, and the higher the value of discrimination score the more likely the specimen is a lung cancer. Also, any new case of lung condition with a Discriminant Score of $<0$ is considered as a benign condition, and the lower the value of Discrimination score the more likely the specimen is a benign condition.

\section{Recommendations:-}

Further molecular studies with larger sample sizes are suggested to be conducted, and with large number of genes to reinforce the results of current study. Further molecular studies on non-invasive samples such as saliva, body fluids and/or blood to design a discrimination score (D score) for screening of other internal malignancies such as cancers of gastro-intestinal tract are recommended. 


\section{References:-}

1. Rebecca Siegel, Deepa Naishadham, and Ahmedin Jemal. Cancer statistics 2013. CA: A Cancer Journal for Clinicians; January2013, 63(1): 11-30.

2. Al Hasnawi., S.M., et al. (2009). Cancer in Iraq: Distribution by primary tumor site. The new Iraqi Journal of Medicine, 5 (1), pp. 5 - 8 .

3. Jiang Y, Jin P, Chang RS, Yao SX, Zhou QH, Fan YG, and Qiao YL. Analysis of sensitivity and specificity of sputum cytology screening for lung cancer with different positive criteria]. Zhonghua Yu Fang Yi Xue Za Zhi. $2011 \mathrm{Jul} ; 45$ (7): 605 - 8.

4. Mitul B Modi, Mitesh R Rathva, Nupur R Shah, Manasi Trivedi and Harshad Patel. Role of FNAC in Lung Carcinoma and its Histo-Cytological Correlation. J Lung Pulm Respir Res 3(4):00090.

5. Marco Andolfi, Rossella Potenza, Rosanna Capozzi, Valeria Liparulo, Francesco Puma, and Kazuhiro Yasufuku. The role of bronchoscopy in the diagnosis of early lung cancer: a review. J Thorac Dis. Nov 2016; 8(11): $3329-37$.

6. Doina-Ecaterina Tofolean, Ariadna-Peronela Fildan, Elena Dantes, Laura Mazilu, E. Dumitru, and Liliana Tuta. The Importance of Bronchoscopy in Early Lung Cancer (LC) Diagnosis. The Journal of "Ovidius" University of Constanta (2017) 22 (8): 270 -77.

7. Bert Gold, Milena Cankovic, Larissa V. Furtado,Frederick Meier, and Christopher D. Gocke. Do Circulating Tumor Cells, Exosomes, and Circulating Tumor Nucleic Acids Have Clinical Utility? Journal of Molecular Diagnosis. May 2015; 17(3): 209-24. doi: 10.1016/j.jmoldx.2015.02.001.

8. Schwarzenbach H1, Hoon DS, and Pantel K. Cell-free nucleic acids as biomarkers in cancer patients. Nat Rev Cancer (June 2011);11(6):426-37. doi: 10.1038/nrc3066. Epub 2011 May 12.

9. Xiao Han, Junyun Wang, and Yingli Sun. Circulating Tumor DNA as Biomarkers for Cancer Detection. Genomics, Proteomics \& Bioinformatics (April 2017); V 15 (2): 59-72.

10. Akira Mogi and Hiroyuki Kuwano. TP53 Mutations in Non-Small Cell Lung Cancer. Journal of Biomedicine and Biotechnology, 2011Volume (2011), Article ID 583929, 9 pages.

11. Vähäkangas, K.H., Bennett, W.P., Castrén, K., Welsh, J.A., Khan, M.A., Blömeke, B., Alavanja, M.C., \& Harris, C.C. (2001). p53 and K-ras mutations in lung cancers from former and never-smoking women. Cancer Res, 61(11), pp. 4350-6.

12. Peter M. K. Westcott1,3 and Minh D. To. The genetics and biology of KRAS in lung cancer. Chin J Cancer. $2013 \mathrm{Feb} ; 32(2)$ : 63-70.

13. Eun Young Kim, Arum Kim, Se Kyu Kim, and Yoon Soo Chang. MYC expression correlates with PD-L1 expression in non-small cell lung cancer. Lung Cancer; August 2017, vol 110; 63 - 67.

14. Ádám Nagy, Lörinc Sándor Pongor, András Szabó, Mariacarmela Santarpia, and Balázs Győrffy. KRAS driven expression signature has prognostic power superior to mutation status in non-small cell lung cancer. Int $\mathbf{J}$ Cancer. (Feb 2017); 140(4): 930-37.

15. Fazli Wahida, Adeeb Shehzada, Taous Khanb, and You YoungKim. MicroRNAs: Synthesis, mechanism, function, and recent clinical trials. Biochimica et Biophysica Acta (BBA) - Molecular Cell Research; November 2010, Volume 1803(11); 1231-1243.

16. Yang Y, Meng H, Peng Q, Yang X, Gan R, and Zhao L et al. Downregulation of microRNA-21 expression restrains non-small cell lung cancer cell proliferation and migration through upregulation of programmed cell death.

17. Xue X, Liu Y, Wang Y, Meng M, Wang K, and Zang X et al. MiR-21 and MiR-155 promote non-small cell lung cancer progression by downregulating SOCS1, SOCS6, and PTEN. Oncotarget; Dec 2016, 7(51): 84508 84519.

18. Shi Y, Liu C, Liu X, Tang DG, and Wang J. The microRNA miR-34a inhibits non-small cell lung cancer (NSCLC) growth and the CD44hi stem-like NSCLC cells. PLoS One; March 2014, 9(3). doi: 10.1371/journal.

19. Yu Y, Zuo J, Tan Q, Zar Thin K, Li P, and Zhu M et al. Plasma miR-92a-2 as a biomarker for small cell lung cancer. Cancer Biomark; 2017;18(3):319 - 327. doi: 10.3233/CBM-160254.

20. Du L1, Schageman JJ, Subauste MC, Saber B, Hammond SM, and Prudkin L et al. miR-93, miR-98, and miR197 regulate expression of tumor suppressor gene FUS1. Mol Cancer Res. Aug 2009;7(8):1234-43. doi: 10.1158/1541-7786.

21. mirVana $^{\mathrm{TM}}$ miRNA Isolation Kit, with phenol. Retrieved online from; https://www.thermofisher.com/order/catalog/product/AM1560 on 2nd of March 2017.

22. High-Capacity RNA-to-cDNA ${ }^{\mathrm{TM}}$ Kit protocol from applied Biosystem. Retrieved online March 2017 from: https://www.thermofisher.com/order/catalog/product/4387406.

23. Applied Biosystems ${ }^{\mathrm{TM}}$ SYBR $^{\mathrm{TM}}$ Green PCR Master Mix. Retrieved online at May 2017 from: 
https://www.fishersci.com/shop/products/applied-biosystems-sybr-green-pcr-master-mix-6/p-4927004.

24. Barber RD, Harmer DW, Coleman RA, and Clark BJ. GAPDH as a housekeeping gene: analysis of GAPDH mRNA expression in a panel of 72 human tissues. Physiol Genomics, May 2005; 21(3): 389-95.

25. Applied Biosystems ${ }^{\mathrm{TM}} \mathrm{TaqMan}^{\mathrm{TM}}$ Universal Master Mix II, No UNG. Retrieved online at may 2017 from:https://www.fishersci.com/shop/products/taqman-universal-mmix-ii/4440040.

26. Alexandra Rogue, Emilie Floch, Roy Forster and Nick Pearson. miRNA quantification and evaluation of suitable housekeeping genes in the major pre-clinical species. Safety and Health Research Laboratories. Retrieved online at May 2017 from: https://www.citoxlab.com/wp-content/uploads/2014/07/ miRNAquantification-and-evaluation-of-suitable-housekeeping-genes-in-the-major-pre-clinical-species.pdf.

27. MxPro QPCR Software Instruction Manual for Mx3000P and Mx3005P QPCR Systems. Retried online at $6^{\text {th }}$ of January 2017 from; https://arboretum.harvard. edu/wp-content/uploads/Mx3000P_and_ Mx3005P_User_Guide1.pdf.

28. Using SPSS for item analysis retrieved online at March 2017 from: https://www. google.iq/search?source=hp\&ei=jeVJWs2bFMjKsAfzgo3AAQ\&q=Using+SPSS+for+item+analysis\&oq=Usin g+SPSS+for+item+analysis\&gs_l=psy-ab.3... 6111.6111.0.7866.1.1.0.0.0.0.0.0..0.0.... ...1c.2.64.psyab..1.0.0....0. Tq6bukk4Txg.

29. IBM SPSS Statistics v24 Free download. Retrieved online at $25^{\text {th }}$ of June 2017 from: https://rahim-soft.org/ibmspss-statistics-v24-free-download/.

30. Calculating Difficulty, Discrimination and Reliability Index/Standard Error of Measurement. Retrieved online at $4^{\text {th }}$ of March 2017 from: https://ppukm.org/2015/04/02/calculating-omr-indexes/.

31. Discrimination | Item Analysis - Sites at Penn State. Retrieved online at $4^{\text {th }}$ of March 2017 from http://sites.psu.edu/itemanalysis/discrimination/.

32. Al-Alusi F. Lung cancer in Iraq, the trend in the decade (1986-1995)-A clinicopathological study. J Fan Med Baghdad, 2002, 44, pp. 175-178.

33. Menkhi, A. Molecular study in lung cancer for detection of RB tumor suppressor gene mRNA and c-MYC, Her-2/neu, K-RAS oncogenes. PhD thesis, Baghdad College of Medicine, 2011.

34. Saeed R M. P53 overexpression in lung cancer - A histopathological study FICMS. Pathol. Thesis 2007, Baghdad College of medicine.

35. Thabit MA. Changing pattern of lung cancer: a pathological and immunohistochemical- A histopathological study FICMS. Pathol. Thesis, 2001, Baghdad College of Medicine.

36. Kersting CF, Kraus A \& Behn M. Differential Frequencies of p16INK4a Promoter Hypermethylation, p53 Mutation, and K-ras Mutation in Exfoliative Material Mark the Development of Lung Cancer in Symptomatic Chronic Smokers. Journal of Clinical Oncology, 2000, 18(18), pp. 3221-3229.

37. Abdul-Majeed BA. P53 mRNA in- Situ Hybridization analysis and Immunohisto chemical Expression in Lung Cancer: A Comparative Study. Journal of the Faculty of Medicine, 2010, 52(3), pp. 370-377.

38. Paone, G. Discriminant analysis on small cell lung cancer and non-small cell lung cancer by means of NSE and CYFRA-21.1. Euro Respiratory Journal, 1995, 8, pp. 1136-1140.

39. Marchevsky, A.M. Classification of individual lung cancer cell lines based on DNA methylation markers: use of linear discriminant analysis and artificial neyral networks. J Mol Diagn, 2004, 6(1), pp. 28-36.

خلاصة البحث باللغة العربية

الخلفية:

سرطان الرئة سبب رئيسي للوفاة في جميع انحاء العالم. معظم الحالات عند التشخيص تكون قام وصلت التى مراحل متقدمة ومن غير المثكن

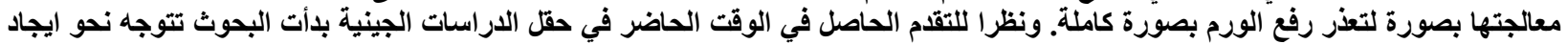

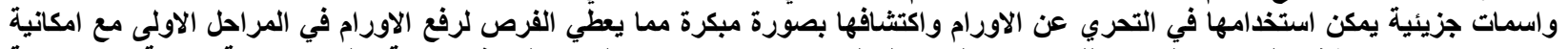

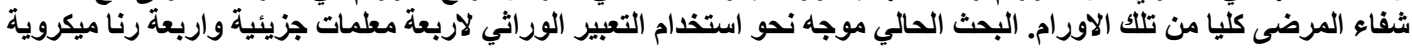

وذلك لكون تلك الفحوصات تجرى على نماذج من مصول الام ولاتحتاج الى استعمال نوظير القصبات او الى التخدير العام مما يسبب مضاعفات للمرضى وخصوصا معظمهم من كبار السن ولايهم مشاكل صحية مزمنة.

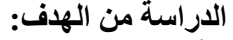

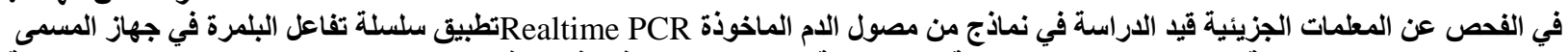

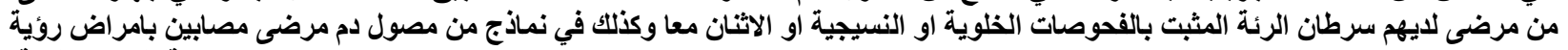
مزمنة غير سرطانية.

أيجاد معادلة حسابية يمكن بواسطتها الكثف عن سرطانات الرئة عن طريق اخذ عينات دم من المرضى وخاصة المرضى المعرضين للاصابة بسرطان الرئة مثل المدخنين وات دمن العمال في المصاتع وخاصة معامل الاسبست. 
المرضى وطرائق العمل: البحث هو دراسة تجريبية شملت ستون حالة مرضية من المرضى المراجعين لقسم جراحة الصدر في مستثفى اليرموك

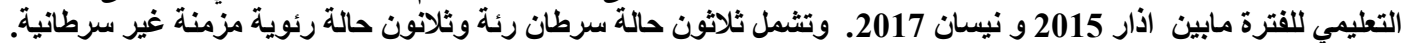

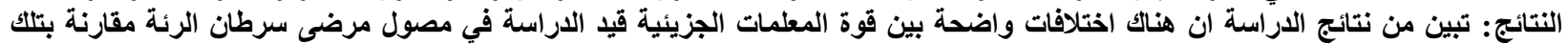

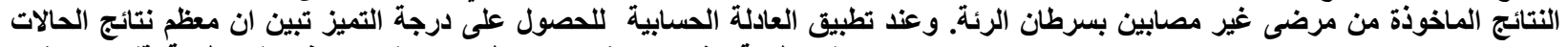

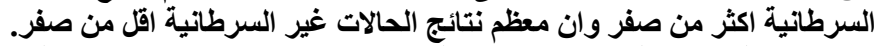

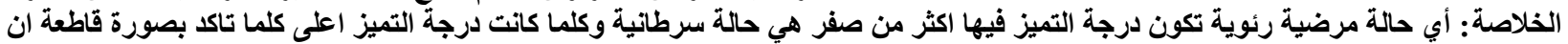

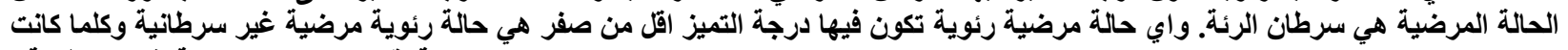
القيمة اقل كلما تاكد ان الحالة غير سير سرطانية.

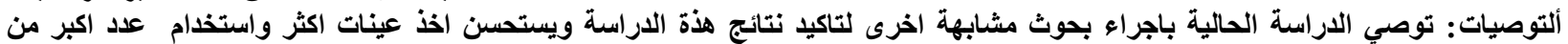

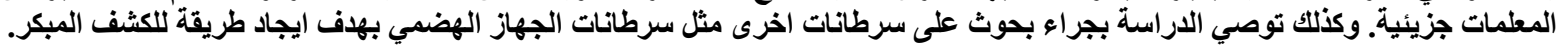

